\title{
Clinical Study on Wuling Powder and Modified Shengmai Powder Treating Chronic Cardiac Insufficiency
}

\author{
Youfu Ke ${ }^{1, *}$, Chun Xiang $\mathrm{Li}^{2}$, Junxian Zheng ${ }^{3}$ \\ ${ }^{1}$ The University of Hong Kong, School of Chinese Medicine, Hong Kong, 999077, China \\ ${ }^{2}$ The Second Affiliated Hospital of Fujian Medical University, Quanzhou, 362000, China \\ ${ }^{3}$ Zhejiang Academy of Chinese Medicine, Hangzhou, 310007, China \\ *Corresponding Author: keyoufu@gmail.com
}

Copyright $@ 2013$ Horizon Research Publishing All rights reserved.

\begin{abstract}
Objective: To evaluate the efficacy and safety of Wuling Powder and Modified Shengmai Powder on treating chronic cardiac insufficiency. Methods: 42 cases of chronic cardiac insufficiency patients with heart function ranging from NYHA class II to IV took Wuling Powder and Modified Shengmai Powder for 2 weeks to treat chronic cardiac insufficiency without changing former medication. NYHA heart function, systolic pressure, diastolic pressure, heart rate, and 6-minute walk test were evaluated before and after treatment. Results: Wuling Powder and Modified Shengmai Powder decreased systolic pressure, diastolic pressure, heart rate, and increased the 6-minute walk distance significantly $(\mathrm{P}<0.01)$. Total effective rate was 92.9\%. There was no serious side effect. Conclusion: Using samples from outpatients, this study shows Wuling Powder and Modified Shengmai Powder is effective and safe to treat chronic cardiac insufficiency.
\end{abstract}

Keywords Wuling Powder, Shengmai Powder, Chronic Cardiac Insufficiency, Chronic Heart Failure, Congestive Heart Failure

Fund Project: Zhejiang Science and Technology Department Fund No.2008F3036

\section{Introduction}

Chronic cardiac insufficiency (CCI) is a progressive disease with significant morbidity and mortality. Many of the evidence-based therapies for heart failure provide symptomatic benefit. There is evidence of varying strengths for pharmacologic and nonpharmacologic relief of common symptoms like dyspnea, fatigue, pain, and depression[1]. Despite angiotensin-converting enzyme inhibitors, angiotensin II receptor blocker, $\beta$-blockers, diuretics, cardiac resynchronization therapy and implantable cardioverter-defibrillator used as the therapeutic approaches, CCI still need to search alternative and complementary treatment[2].
Chinese herbal medicine has been used to treat CCI for over 2000 years. Wuling Powder induces diuresis, excretes liquids and eliminates edema[3]. Diuretics are regarded as the first-line treatment for $\mathrm{CCI}$, which reduces the risk of death and heart failure aggravation compared to placebo[4]. Shengmai Powder can strengthen cardiac contractility, improve patient's quality of life, effective in ventricular remodeling[5]. Both Wuling Powder and Shengmai Powder aim at pathogenesis of CCI. We hypothesize that it is beneficial to use both of them to treat CCI.

In western medicine, it is common practice for patients who have already used one or several kinds of anti-heart failure therapy to add on another kind of medication to futher improve heart function. The add-on effect of the added drug is still considered to be effective if chronic cardiac insufficiency finally gets improved[6].

So we hypothesize that it is beneficial and convenient to use both Wuling Powder and Shengmai Powder as an open add-on therapy for treating CCI and it can still demonstrate whether it is effective or not even without setting up control group.

To evaluate the efficacy and safety of Wuling Powder and Shengmai Powder on treating CCI, we collected 42 cases of CCI patients with heart function ranging from NYHA class II to IV to have taken Wuling Powder and Modified Shengmai Powder for 2 weeks to treat CCI without changing former medication. Here is our report.

\section{Material and Methods}

\subsection{Subject of Study}

42 cases of CCI patients with heart function ranging from NYHA class II to IV were included. With 22 male and 20 female cases, the average age was $64.3 \pm 13.6$ years. 9 cases belonged to heart function class II; 23, III and 10, IV. 32 cases suffer from hypertensive heart disease; 19, coronary heart disease (15, both hypertensive heart disease and coronary heart disease); 3, rheumatic heart disease; 2, pulmonary heart disease; 1 , primary cardiac myopathy. 
The patients were outpatients who came to consult Chinese medicine practitioner for better control. They were willing to administer Chinese Medicine as an open add-on therapy. So no one withdrew.

\subsection{Treatment Methods}

All CCI patients took Wuling Powder and Modified Shengmai Powder for 2 weeks without changing former medication. Formula: Poria 15 grams, Polyporus 15 grams, Rhizima Atractylodis Macrocephalae 15 grams, Rhizoma Alismatis 15 grams, Ramulus Cinnamomi 10 grams, Radix Ginseng 10 grams, Radix Ophiopogonis 10 grams, Fructus Schisandrae Chinensis 10 grams, Radix Notoginseng 10 grams, Radix Astragali 30 grams, Radix Rehmanniae 30 grams. One dose a day, simmer gently for about 35 minutes to produce about $400 \mathrm{ml}$ decoction, oral administration two times daily.

NYHA heart function, systolic pressure(SP), diastolic pressure(DP), heart rate (HR), and 6-minute walk test $(6 \mathrm{MWT})$ were evaluated before and after treatment.

\subsection{Criterion of Therapeutical Effect on Heart Function}

The criterion is exactly based on "Guideline for clinical research on new drug of Chinese Medicine treating heart failure" which forms one chapter of a book called "Guidelines for clinical research on new drug of Chinese Medicine" compiled by State Food and Drug Administration of China[7]. Markedly effective: NYHA heart function classification returned to I or was improved two classes. Effective: NYHA heart function classification was improved one class. Ineffective: NYHA heart function classification had no change or deteriorated. Total effective rate was the combination of markedly effective rate and effective rate.

\subsection{Indexes Measurement}

NYHA heart function classification, 6-minute walking test (6MWT), heart rate (HR), SP, DP were evaluated before and after treatment according to methods provided by the book and guidelines[6,7]. NYHA heart function classification, 6MWT were evaluated during 8:00-12:00 am; HR, SP, DP were measured by electronic measuring device two times a day (8:00-9:00 am and pm), taking the mean values.

\subsection{Statistics}

Paired sample T-test was used to analyze SP, DP, HR and 6MWT differences between pre-treatment and post treatment.

\section{Results}

\subsection{Changes in Blood Pressure, Heart Rate and}

\section{6-minute walk test (Table 1)}

Wuling Powder and Modified Shengmai Powder decreased the CCI patients' systolic pressure, diastolic pressure, heart rate, and increased the 6-minute walk distance significantly $(\mathrm{P}<0.01)$.

Table 1. Changes in SP,DP, HR,6MWT ( $\bar{X} \pm \mathrm{S})$

n $\mathrm{SP}(\mathrm{mmHg}) \mathrm{DP}(\mathrm{mmHg}) \mathrm{HR}(\mathrm{bpm}) 6 \mathrm{MWT}(\mathrm{m})$

pre-treatment $42147 \pm 9.585 \pm 8.2 \quad 89 \pm 8.4368 \pm 54$

post treatment $42124 \pm 8.8 * 72 \pm 7.3 * \quad 70 \pm 7.1 * \quad 488 \pm 72 *$

*compared with pre-treatment $\mathrm{P}<0.01$

\subsection{The Efficacy of Wuling Powder and Modified Shengmai Powder on Treating CCI(Table 2)}

After taking Wuling Powder and Modified Shengmai Powder for 2 weeks without changing their former medication, markedly effective rate and effective rate showed $40.5 \%$ and $52.4 \%$ respectively. Hence, total effective rate was $92.9 \%$.

Table 2. Case Numbers of Therapeutical Effect and Total Effective Rate $\mathrm{n}$ markedly effective effectiveineffective total effective rate

$\begin{array}{lll}421722 & 3 & 92.9 \%\end{array}$

\subsection{The Safety of Wuling Powder and Modified Shengmai Powder on Treating CCI}

4 cases of CCI patients with severe leg and ankle edema reported urinary frequency and/or nocturia at the beginning of the treatment, after several days of treatment, when severe leg and ankle edema were gone, it disappeared, suggesting it result from pharmacological action of Wuling Powder inducing diuresis, excreting liquids and eliminating edema. There was no other severe side effects such as dry mouth, insomnia, feverish dysphoria, tidal fever, tachycardia, palpitation, dizziness, gastrointestinal discomfort, and serum electrolyte disturbance etc.

\section{Discussion}

Chinese herbal medicines have been used to treat CCI effectively for over 2000 years. In TCM theory, pathogenesis of CCI is due to heart Yangqi deficiency, blood stasis and fluid retention. Yangqi can promote water metabolism and accelerate blood circulation, acting as the vital elements for human body to maintain life activity. So invigorating Yangqi, promoting blood circulation to remove blood stasis, using diuretic to alleviate water retention are TCM therapeutic principles in treating CCI.

Wuling Powder, consisting of Poria, Polyporus, Rhizima Atractylodis Macrocephalae, Rhizoma Alismatis, Ramulus Cinnamomi, is one of the important decoction in the Treatise on Exogenous Febrile Diseases and a commonly used formula, which has the function of inducing diuresis, 
excreting liquids and eliminating edema. Ramulus Cinnamomi can lower blood pressure, reinforce cardiac contractility, reduce blood-lipid and blood sugar[8,9]. One experimental study in rats shows wuling powder has satisfying therapeutic effects on increasing the discharge of urine, decreasing the blood pressure and keeping the balance of the serum electrolyte with renal hypertension[3].

Shengmai Powder, consisting of Radix Ginseng, Radix Ophiopogonis, Fructus Schisandrae Chinensis, was described by the famous physician Li Gao (1180-1251), who was one of the founders of the "Spleen/Stomach" school concerning with treating qi-deficiency syndromes. It is administered to patients suffering from a serious illness, deficient in qi and yin, especially chronic cardiac insufficiency, heart attack. Shengmai means "generating pulse," indicating that it is given to persons with a very weak pulse. Radix Ginseng invigorates qi, Radix Ophiopogonis nourishes yin, and Fructus Schisandrae Chinensis astringes sweating, generates fluids, and helps Radix Ophiopogonis nourish yin. It can regulate the heart rate, increase heart output, strengthen cardiac contractility, reduce oxygen consumption in the heart muscles, adjust blood pressure, exert actions against shock, expand the coronary arteries and increase coronary arterial blood flow. It can be used to treat coronary heart disease, pulmonary disease, rheumatic heart disease, arrhythmia, heart neurosis, hypotension, sunstroke[5] etc.

In clinical practice, in order to treat CCI more effectively, some other herbs may be added to Shengmai Powder to enhance cardiac contractility, endow with blood pressure lowering effect to reduce cardiac overload, accelerate blood circulation to dissipate blood stasis, and so on. Since Radix Notoginseng[10,11], Radix Astragali[12,13] and Radix Rehmanniae[14]can strengthen cardiac contractility, decrease blood pressure, reduce blood sugar and fat, activate blood circulation to dissipate blood stasis, we modified Shengmai Powder and added these three herbs to reinforce the formula's efficacy.

In our clinical research, both Wuling Powder and Modified Shengmai Powder were used for invigorating heart Yangqi, strengthening cardiac contractility, promoting blood circulation to remove blood stasis, promoting urination to alleviate water retention, decreasing blood pressure, and reducing cardiac overload, thus counteracting pathogenesis of CCI.

It is unethical to give placebo or no treatment to $\mathrm{CCI}$ patients, especially to those with elevated blood pressure. The add-on effects of Wuling Powder and Modified Shengmai Powder to existing treatments have demonstrated that Wuling Powder and Modified Shengmai Powder were useful to improve heart function and control blood pressure in CCI patients.

\section{Conclusion}

With Wuling Powder strengthening cardiac contractility, increasing urinary volume, decreasing blood pressure without causing the serum electrolyte imbalance, and together with Modified Shengmai Powder reinforcing cardiac contractility, lowering blood pressure, and accelerating blood circulation to remove blood stasis, treating CCI with Wuling Powder and Modified Shengmai Powder is effective and safe.

\section{REFERENCES}

[1] Goldfinger JZ, Adler ED. End-of-life options for patients with advanced heart failure. Curr Heart Fail Rep. 2010;7(3):140-147.

[2] Mant J, Al-Mohammad A, Swain S, Laramée P; Guideline Development Group. Management of chronic heart failure in adults: synopsis of the National Institute For Health and clinical excellence guideline. Ann Intern Med. 2011;155(4):252-259.

[3] Han YP, Wang NS, Mi SQ, et al. Effect of Wuling Powder on rats with renal hypertension. J Chin Integr Med. 2003; 1(4): 285-288.

[4] Faris RF, Flather M, Purcell H, Poole-Wilson PA, Coats AJ. Diuretics for heart failure. Cochrane Database Syst Rev. 2012;2:CD003838

[5] Gu YM, Ye SL Sun Y, et al. Effect of Shengmai Powder on Ventricular Remodeling and Quality of Life of Chronic Heart Failure Patients. Journal of Traditional Chinese Medicine. 2009;50( 2):127-129.

[6] Chen HZ, Lin GW. Practice of internal medicine.13th Ed. Beijing :People,,s Medical Publishing House.2009.1349-170 8

[7] Zheng XY. Guidelines for clinical research on new drug of Chinese Medicine.Beijing:China Medical and Pharmaceutical Science and Technology Publishing House, $2002: 73--85$.

[8] Liu P, Zhang LP. Studies on Chemical Constituents in Dried Tender Stem of Cinnamomi Cassia. Liaoning Journal of Traditional Chinese Medicine.2012;39(10):1926-1927.

[9] Verspohl Ej, Bauer K, Neddemann E. Antidiabetic effect of Cinnamomum cassia and Cinnamomum zeylanicum in vivo and in vitro.Phytother Res.2005;19( 3) : 203-206.

[10] Ng TB. Pharmacological activity of sanchi ginseng (Panax notoginseng). J Pharm Pharmacol. 2006 ;58(8):1007-1019.

[11] Shang Q, Xu H, Liu Z, Chen K, Liu J. Oral Panax notoginseng Preparation for Coronary Heart Disease: A Systematic Review of Randomized Controlled Trials. Evid Based Complement Alternat Med. 2013:940125.

[12] Zhang Q, Gao WY, Man SL. Chemical composition and pharmacological activities of Astragali Radix. China journal of Chinese Materia Medica.2012;37(21):3203-3207

[13] Cheng Y, Jiang SS. Cardiovascular protective effects of astragaloside IV. J Med Postgra.2011;24(6):637-640

[14] Li J, Zhang LP, Zhang ZL. Recent development of Radix rehmanniae. Journal of Henan University of Chinese Medicine.2005;20(6):79-83. 